\title{
ИССЛЕДОВАНИЕ ЗАВИСИМОСТИ СВОЙСТВ ПОВЕРХНОСТНО- АКТИВНЫХ ВЕЩЕСТВ ОТ ИХ ХИМИЧЕСКОЙ СТРУКТУРЫ
}

\section{1. ИОННАЯ КЛАССИФИКАЦИЯ}

(Представил О. Киррет)

При классификации поверхностно-активных веществ (ПАВ) исходят из структуры [1] и свойств [ $\left.{ }^{2}\right]$, а также из методов синтеза [ $\left.{ }^{3}\right]$ и применения [ $\left.{ }^{4}\right]$ этих соединений [5]. Наиболее употребительной является классификация, основанная на ионном поведении ПАВ в водном растворе. В статье сделана попытка развить эту классификацию.

\section{1. Действующая классификация ПАВ}

Классификация ПАВ по ионному характеру [2] гидрофильной части молекулы основана на определенной зависимости свойств ПАВ от их ионного поведения в растворе. По типу ионной формы полярных групп поверхностно-активной части молекулы в растворе ПАВ делят на два класса: ионогенные и неионогенные. Первые имеют функциональные группы, которые в водном растворе ионизируются, образуя положительно (катионные ПАВ) или отрицательно (анионные ПАВ) заряженные поверхностно-активные ионы, или же их заряд зависит от рН среды (амфотерные ПАВ). Неионогенные ПАВ содержат полярные недиссоциирующие группы.

Действующая классификация подразделяет ПАВ по ионному поведению в водном растворе следующим образом:

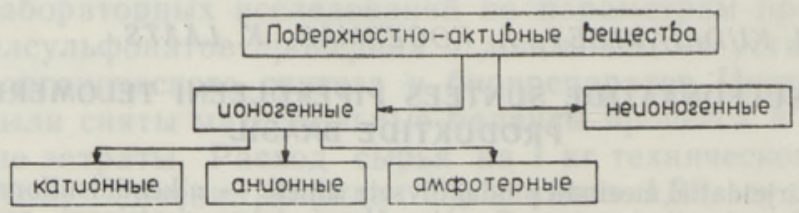

Следовательно, класс ПАВ определяется способностью полярной группы молекулы ПАВ ионизироваться и типом заряда ионизированной формы молекулы. В таком случае гидрофильные группы классифицируются на три типа (C, A и $\mathrm{N})$, которые и определяют три основных класса ПАВ:

1. Катионный (C), например, $-\stackrel{+}{\mathrm{N}} \mathrm{H}_{3} \rightleftharpoons-\mathrm{NH}_{2}+\mathrm{H}^{+},-\stackrel{+}{\mathrm{N}} \mathrm{H}_{2}-\rightleftharpoons-\mathrm{NH}-+\mathrm{H}^{+},>\stackrel{+}{\mathrm{N}} \mathrm{H}-\rightleftharpoons>\mathrm{N}-+\mathrm{H}^{+}$,

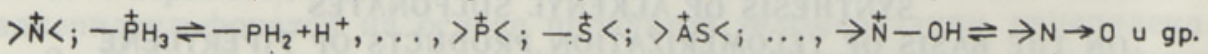

2. Анионный (A), например, $-\mathrm{COOH} \rightleftharpoons-\mathrm{COO}^{-}+\mathrm{H}^{+},-\mathrm{O}-\mathrm{OH} \rightleftharpoons-\mathrm{O}-\mathrm{O}^{-}+\mathrm{H}^{+},-\mathrm{SO}_{3} \mathrm{H}=$ $-\mathrm{SO}_{3}^{-}+\mathrm{H}^{+},-\mathrm{SO}_{2} \mathrm{NH}-\rightleftharpoons-\mathrm{SO}_{2} \mathrm{~N}^{-}-+\mathrm{H}^{+},-\mathrm{OP}(=0)(\mathrm{OH}) \mathrm{O}-\rightleftharpoons-\mathrm{OP}(=0)-\left(0^{-}\right) \mathrm{O}^{-}+\mathrm{H}^{+}$ u gp.

3. Неионогенный (N), например, $-\mathrm{OH},-\mathrm{O}-,-\mathrm{CONH}-$ u gp. 
В принципе возможны следующие комбинации из этих классов ПАВ: CA - амфотерные, CN - катионно-неионогенные, AN - анионно-неионогенные, CAN - амфотерно-неионогенные.

В настоящее время из этих би- и трифункциональных комбинаций возведены в ранг класса только амфотерные ПАВ, в молекулах которых имеются в интервале $\mathrm{pH} \mathrm{0-14}$ (практически наиболее используемом) противоположно заряженные группы. Остальные комбинации в качестве самостоятельных классов не признаны, по-видимому, исходя из предположения, что введение в молекулу ПАВ одной неионогенной группы или нескольких, как и добавление к уже существующей ионогенной группе подобной не является достаточным условием для существенного изменения свойств (класса) ПАВ. Поэтому ПАВ типа $\mathbf{C N}$ и $\mathbf{A N}$ причисляются к классам С и A соответственно. Аналогично поступают с ПАВ типа $\mathbf{C}_{m}$ и $\mathbf{A}_{n}$, где $m, n>1$.

В рамках действующей классификации по ионному состоянию можно все ПАВ представить общей формулой

$$
\mathrm{R} \mathbf{C}_{m} \mathbf{A}_{n} \mathrm{~N}_{r},
$$

где $\mathrm{R}$ - гидрофобная часть молекулы ПАВ, C, A, N - типы гидрофильных групп, $m, n, r \geqslant 0$.

\section{2. Определение типа гидрофильных групп по их способности к диссоциации}

Изучение катионного и анионного типов ионогенных функциональных групп показало, что они, в свою очередь, могут быть подразделены на два типа. К первому типу относятся группы, способные в результате протолиза в интервале $\mathrm{pH} 0-14$ полностью переходить в другую ионную форму: к примеру, первичная, вторичная и третичная аминогруппы и карбоксильная группа. K второму типу относятся те группы, ионный заряд которых остается в этих пределах величин $\mathrm{pH}$ неизменным, поскольку их величины $\mathrm{pK}_{a}$ выходят за пределы $\mathrm{pH} 0-14$ или же ионные переходы запрещены молекулярной структурой. Примерами катионоактивных веществ этого типа являются положительно заряженные груп пы ониевых соединений. Из них четвертичные аммониевые, фосфониевые и третичные сульфониевые известны как группы катионных и амфотерных ПАВ. Примерами анионных групп, сохраняющих заряженную группу в интервале $\mathrm{pH} 0-14$, могут быть сульфогруппа $\left(\mathrm{pK}_{a}<0-1\right)$, гидроксильные группы 2-гидроксихинолина и 1-гидроксиизохинолина, т. е. сильнокислотные группы, диссоциация которых полностью подавляется только при $\mathrm{pH}<0$.

Определим тип зависимости ионного состояния гидрофильной группы от рН среды как ее характеристику диссоциации (ХД), причем ХД молекулы складывается аддитивно от ХД всех ее групп. Тогда, учитывая вышеизложенное, можно все гидрофильные группы разбить на пять типов:

1. Катионные в заряженной ионной форме $\left(\mathrm{C}^{\prime}\right)$, например,

$$
>_{N}^{+}<,>_{P}^{+}<,>\stackrel{S}{S}-^{+}-\mathbb{N}_{+}^{N}=.
$$

2. Катионные, выступающие в двух ионных формах (C), например,

$$
\left.\left.-\stackrel{+}{\mathrm{N}} \mathrm{H}_{3} \rightleftharpoons-\mathrm{NH}_{2}+\mathrm{H}^{+} \text {u }-R_{\mathrm{NH}_{2}}^{\mathrm{N}}\right]=-\mathrm{NH}_{\mathrm{NH}}^{\mathrm{N}}\right]+\mathrm{H}^{+}(\mathrm{pK} a=0-14) .
$$


3. Анионные, выступающие в интервале $\mathrm{pH} 0-14$ в заряженной ионной форме $\left(\mathrm{A}^{\prime}\right)$, например,

$$
-\mathrm{SO}_{3} \mathrm{H} \rightleftharpoons-\mathrm{SO}_{3}{ }^{-+} \mathrm{H}^{+} \quad\left(\mathrm{pK}_{a}<0\right) \text {. }
$$

4. Анионные, выступающие в двух ионных формах (A), например,

$$
-\mathrm{COOH}=-\mathrm{COO}^{-}+\mathrm{H}^{+},-\mathrm{O}-\mathrm{OH}=-\mathrm{O}-\mathrm{O}^{-}+\mathrm{H}^{+}\left(\mathrm{pK} \mathrm{K}_{a}=0-14\right)
$$

5. Неионогенные, выступающие в интервале $\mathrm{pH} 0-14$ в незаряженной ионной форме $(\mathrm{N})$, например,

$$
-\mathrm{OH},-\mathrm{O}-,-\mathrm{CONH}-\left(\mathrm{pK}_{a}>14 \text { и/или } \mathrm{pK}_{b}>14\right) \text {. }
$$

Сравнивая ХД всех пяти типов гидрофильных групп (рис. 1), можно видеть, что в интервале $\mathrm{pH} 0-14$ группы типа $\mathrm{C}^{\prime}, \mathrm{A}^{\prime}$ и $\mathrm{N}$ отличаются друг от друга только характером (зарядом) ионной формы, а группы типа $\mathrm{C}$ и $\mathrm{A}$ изменяют ионную форму $\left(\mathrm{C}^{+} \rightleftarrows \mathrm{C}^{0}\right.$ и $\left.\mathrm{A}^{-} \rightleftarrows \mathrm{A}^{0}\right)$. Необходимо отметить, что классифицирование гидрофильных групп, значения $\mathrm{pK}_{a}$ которых близки к пограничным величинам в интервале $0-14$, в опреде-
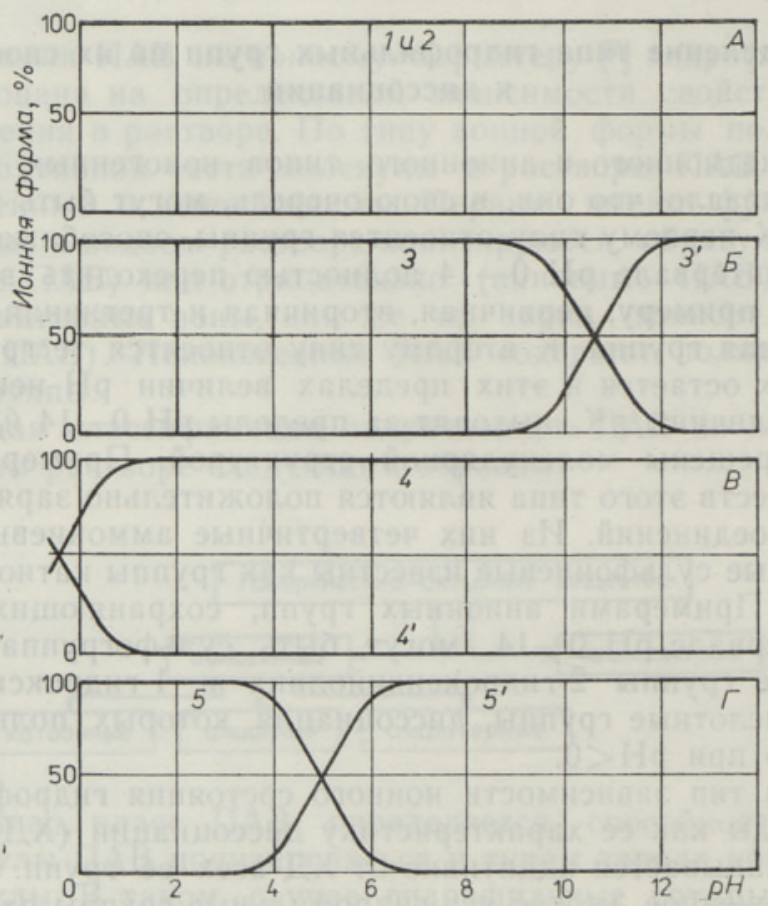

Рис. 1. Характеристики диссоциации гидрофильных групп различного типа. $\mathrm{A}$ - тип $\mathrm{C}^{\prime}$, например аммониевая группа в $\stackrel{+}{\mathrm{R}}\left(\mathrm{CH}_{3}\right)_{3} \mathrm{Cl}^{-}$, и тип $\mathrm{N}$, например гидроксильная и эфирные группы в $\mathrm{RO}\left(\mathrm{CH}_{2} \mathrm{CH}_{2} \mathrm{O}\right)_{r} \mathrm{H}(1,2)$;

Б - тип С, аминогруппа в $\mathrm{C}_{12} \mathrm{H}_{25} \mathrm{NH}_{2}\left(\mathrm{pK}_{a}=10,63\left[{ }^{6}\right]\right), 3-$ форма $\mathrm{C}_{12} \mathrm{H}_{25} \stackrel{+}{\mathrm{NH}_{3}} \cdot \mathrm{X}^{-}$и $3^{\prime}-$ форма $\mathrm{C}_{12} \mathrm{H}_{25} \mathrm{NH}_{2}$;

$\mathrm{B}$ - тип $\mathrm{A}^{\prime}$, сульфогруппа в $\mathrm{RSO}_{3} \mathrm{H}\left(\mathrm{pK}_{a}=-0,33\left[{ }^{7}\right]\right)$, где $\mathrm{R}-$ заместитель, 4 - форма $\mathrm{RSO}_{3}^{-}$и $4^{\prime}$ - форма $\mathrm{RSO}_{3} \mathrm{H}$;

$\Gamma$ - тип $\mathrm{A}$, карбоксигруппа в $\mathrm{C}_{8} \mathrm{H}_{17} \mathrm{COOH}\left(\mathrm{pK}_{a}=4,95\left[{ }^{7}\right]\right), 5-$ форма $\mathrm{C}_{8} \mathrm{H}_{17} \mathrm{COOH}$ н $5^{\prime}$ - форма $\mathrm{C}_{8} \mathrm{H}_{17} \mathrm{COO}^{-}$. катион ${ }^{+}$. 
ленной степени условно, так как при соответствующих значениях $\mathrm{pH}$ группы находятся в стадии перехода в другую ионную форму. Для полного перехода, в количестве, например, 99,99\%, требуется, как известно, изменение $\mathrm{pH}$ среды на 4 единицы.

Действующая классификация относит ПАВ с группами типа $\mathrm{C}^{\prime}$ и $\mathrm{C}$ (по ХД) к катионному классу (C), а ПАВ с группами $\mathrm{A}^{\prime}$ и $\mathrm{A}-$ к анионному классу (A). Такое отнесение нельзя признать обоснованным, так как ХД ПАВ перечисленных типов совершенно различны, а следовательно, различны и их свойства, например зависимость поверхностной активности от $\mathrm{pH}$ среды (рис. 2). Тот факт, что прикладные свойства ПАВ с группами типа $\mathrm{C}^{\prime}$ и $\mathrm{C} \mathrm{A}^{\prime}$ и $\mathrm{A}$ различаются (табл. 1), говорит в пользу выделения их в самостоятельные классы ПАВ.

Особенно заметные различия в свойствах ПАВ с различными ХД обнаруживаются в случае соединений с двумя или с несколькими ионизированными группами, когда вследствие различий в ХД кислотных и основных составляющих групп появляются новые качества - амфолитность и цвиттерионность. Из них «амфолитность» появляется при совмещении групп типа С и $\mathrm{A}$, а «цвиттерионность»- при совмещении групп $\mathrm{C}^{\prime}$ и $\AA^{\prime}$. K цвиттерионным ПАВ причисляются иногда и ПАВ, содержащие комбинацию групп $\mathrm{C}^{\prime}$ и $\mathrm{A}$ (бетаиновые), ввиду того что их основной рабочей формой является цвиттерионная, хотя возможна и катионная.

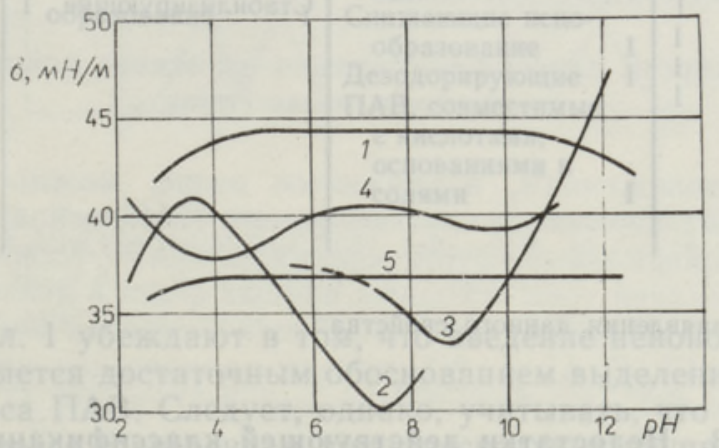

Рис. 2. Зависимость поверхностного натяжения 0,1\%-ных растворов ПАВ с различными характеристиками диссоциации от $\mathrm{pH}$ среды при $16 \pm 1^{\circ} \mathrm{C}\left[{ }^{8}\right]$ (3 при $22^{\circ} \mathrm{C}$, измерена

нами): $\mathrm{C}_{12} \mathrm{H}_{25} \stackrel{+}{\mathrm{O}} . \mathrm{Cl}^{-}$(тип $\mathrm{C}^{\prime}$ ) (1), $\mathrm{C}_{12} \mathrm{H}_{25} \stackrel{+}{\mathrm{N}} \mathrm{H}_{3} \cdot \mathrm{Cl}-$ (тип С) (2), $\mathrm{C}_{12} \mathrm{H}_{25} \mathrm{COONa}$ (тип A) (3), $\mathrm{C}_{12} \mathrm{H}_{25} \mathrm{SO}_{4} \mathrm{Na}$ (тип $\mathrm{A}^{\prime}$ ) (4), $\mathrm{C}_{18} \mathrm{H}_{37} \mathrm{O}\left(\mathrm{CH}_{2} \mathrm{CH}_{2} \mathrm{O}\right)_{7} \mathrm{H}$ (тип $\mathrm{N}$ ) (5).

Следовательно, ХД могут служить показателями, позволяющими упорядочить классифицирование ПАВ с одновременным улучшением соответствия между их классовыми признаками и свойствами. С учетом ХД все ПАВ можно описать общей формулой

$$
\mathrm{RC}_{m}^{\prime} \mathrm{C}_{n} \mathrm{~A}_{p}^{\prime} \mathrm{A}_{q} \mathrm{~N}_{r}
$$

где $\mathrm{C}^{\prime}, \mathrm{C}, \mathrm{A}^{\prime}, \mathrm{A}, \mathrm{N}$ - типы гидрофильных групп по ХД, $m-r \geqslant 0$. 


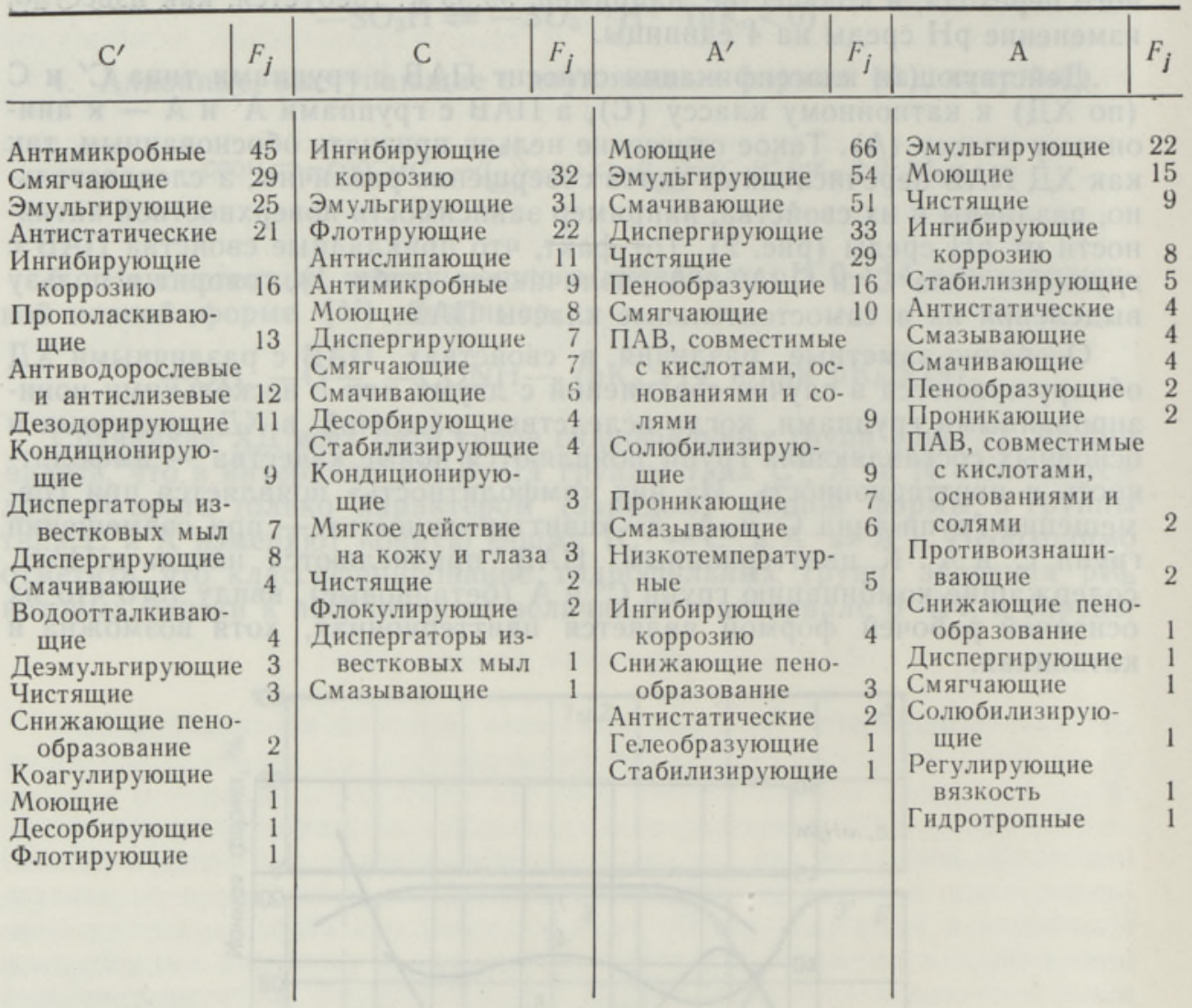

* $F_{j}$ - частота заявления данного свойства.

\section{3. Недостатки действующей классификации}

Проанализируем, какие недостатки присущи действующей классификации и какие возможности предоставляет классификация, построенная на понятиях о ХД.

1. Одно и то же ПАВ можно, в зависимости от рН среды, отнести в различные классы $\left.{ }^{2}\right]$. Например, в щелочной среде алкилкарбоновая кислота полностью диссоциирует и выступает как анионное ПАВ. В кислой среде, где диссоциация карбоксильных групп подавлена, соединение ведет себя как неионогенное ПАВ. В качестве других примеров можно привести алкиламин и окись третичного амина, которые в зависимости от $\mathrm{pH}$ среды могут быть отнесены к классам катионных или неионогенных ПАВ. По новой ХД-классификации эти ПАВ принадлежат к классам А, С и С соответственно.

2. Неионогенные (N) группы в молекулах ионогенных ПАВ, как изменяющий свойства ПАВ фактор, не учитываются. Сравним прикладные свойства ПАВ с группами типа $\mathrm{C}^{\prime}, \mathrm{C}, \mathrm{A}^{\prime}$ и $\mathrm{A}$, с одной стороны, и с группами типа $\mathrm{C}^{\prime} \mathrm{N}, \mathrm{CN}, \mathrm{A}^{\prime} \mathrm{N}$ и $\mathrm{AN}-$ д другой (табл. 1). Выясняется, что с введением неионогенных групп в структуру молекулы ионогенного ПАВ ряды прикладных свойств, характерных для этих классов, существенно перестраиваются, а в некоторых случаях появляются новые специфические свойства или же исчезают прежние. 


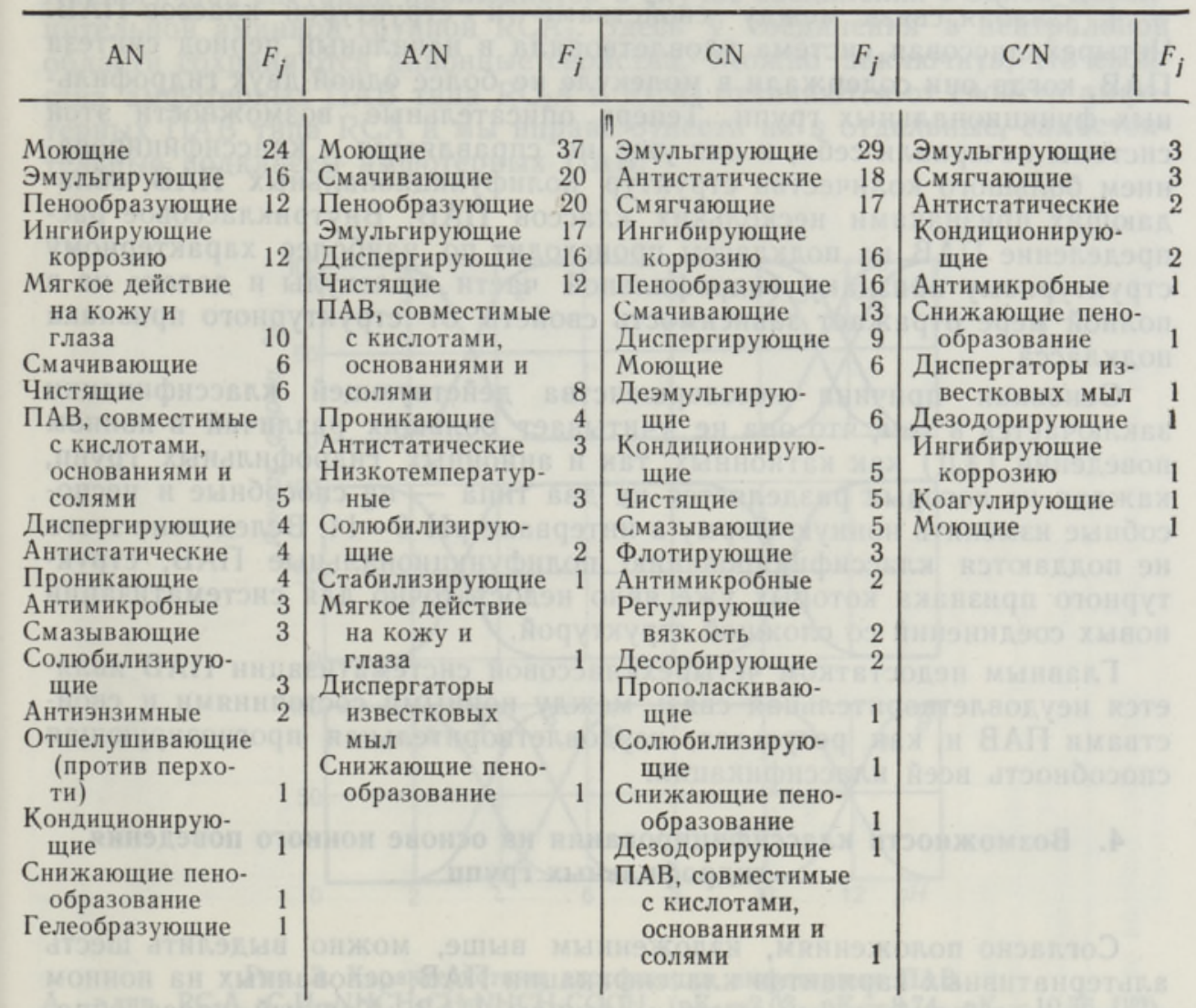

Данные табл. 1 убеждают в том, что введение неионогенной группы в молекулу является достаточным обоснованием выделения нового класса или подкласса ПАВ. Следует, однако, учитывать, что свойства ПАВ заметно изменяются с введением лишь нескольких групп типа $\mathrm{N}$.

3. Рамки действующей классификации слишком узки, чтобы охватить все растущее количество полифункциональных ПАВ, в частности амфотерных. Например,

$$
\underset{\mathrm{RC}}{\mathrm{RN}}(\mathrm{O}) \underset{\mathrm{N}}{\left(\mathrm{CH}_{3}\right) \mathrm{CH}_{2} \mathrm{CH}(\mathrm{OH}) \underset{\mathrm{C}^{\prime}}{\mathrm{CH}_{2}^{\mathrm{N}}}\left(\mathrm{CH}_{3}\right)_{2} \mathrm{CH}_{2} \mathrm{COO}^{-}} \stackrel{\mathrm{A}}{\mathrm{CHC}^{\prime} \mathrm{CAN}}
$$

$$
\begin{aligned}
& \mathrm{RCONHC}_{3} \mathrm{H}_{6} \stackrel{+}{\mathrm{N}}\left(\mathrm{CH}_{3}\right)_{2} \mathrm{CH}_{2} \mathrm{COO}_{-}^{-} \text {. } \\
& \text { RN } \quad C^{\prime} \quad A=R^{\prime} A N
\end{aligned}
$$

В настоящее время их классифицируют как цвиттерионные или бетаиновые ПАВ. Однако свойства этих ПАВ, благодаря наличию в молекуле гидрофильных групп с различными ХД, неидентичны. Так, первое рекламируется как регулирующее вязкость, кондиционирующее, моющее, пенообразующее, смачивающее и чистящее, а второе - как регулирующее вязкость, пенообразующее, чистящее, антимикробное, антистатическое, диспергирующее, стабилизирующее и смягчающее 
кожу. По новой классификации эти ПАВ войдут в классы RC'CAN и $\mathrm{RC} \mathrm{AN}^{\prime}$

4. Слабая связь между свойствами и структурой классов ПАВ. Четырехклассовая система удовлетворяла в начальный период синтеза ПАВ, когда они содержали в молекуле не более одной-двух гидрофильных функциональных групп. Теперь описательные возможности этой системы исчерпали себя, и она уже не справляется с классифицированием большого количества структур полифункциональных ПАВ, обладающих признаками нескольких классов ПАВ. Внутриклассовое распределение ПАВ на подклассы происходит по нанболее характерному структурному признаку гидрофильной части молекулы и далеко не в полной мере отражает зависимость свойств от структурного признака подкласса.

Основная причина несовершенства действующей классификации заключается в том, что она не учитывает больших различий в ионном поведении (ХД) как катионных, так и анионных гидрофильных групп, каждая из которых разделяется на два типа - на способные и неспособные изменять ионную форму в интервале $\mathrm{pH} 0-14$. Вследствие этого не поддаются классифицированию полифункциональные ПАВ, структурного признака которых уже явно недостаточно для систематизации новых соединений со сложной структурой.

Главным недостатком четырехклассовой систематизации ПАВ является неудовлетворительная связь между ионными состояниями и свойствами ПАВ и, как результат, неудовлетворительная прогнозирующая способность всей классификацин.

\section{4. Возможности классифицирования на основе ионного поведения гидрофильных групп}

Согласно положениям, изложенным выше, можно выделить шесть альтернативных вариантов классификации ПАВ, основанных на ионном состоянии гидрофильных групп молекулы ПАВ. Каждый класс образуется комбинацией трех исходных ионных форм в действующей классификации ( $\mathbf{C}, \mathbf{A}, \mathrm{N})$ или комбинацией пяти исходных ионных форм с различными ХД $\left(\mathrm{C}^{\prime}, \mathrm{C}, \mathrm{A}^{\prime}, \mathrm{A}, \mathrm{N}\right)$, причем наличие неионогенной группы в молекуле, как фактор образования нового класса ПАВ, в отдельных вариантах классификации учитывается (варианты III-VI) или не учитывается (варианты I и II). Повторение гидрофильной группы, как аргумент в пользу образования нового класса ПАВ, учитывается в вариантах V и VI. Типы и количество классов по всем возможным вариантам классификации приведены в табл. 2.

Количество классов по вариантам V и VI зависит от количества функциональных групп и вычисляется по уравнению комбинаторики [].

\section{5. Рекомендуемая классификация}

Новая классификация ПАВ по ионному поведению гидрофильных групп учитывает не только все факторы, приведенные в 3-м разделе, но и численный перевес одной группы ноногенного типа над другой в случае амфотерного ПАВ. В качестве примера рассмотрим ХД трех амфотерных ПАВ (рис. 3).

В случае амфотерных ПАВ типа RCA катионные свойства проявляются при $\mathrm{pH} 0-2,5$, а анионные свойства - при $\mathrm{pH} 9,5-14$. В интервале $\mathrm{pH}$ 2,5-9,5 амфотерное ПАВ находится в цвиттерионной форме, его растворимость низкая и технологические свойства практически отсутствуют. С введением в молекулу еще одной группы типа С катионные свойства амфотерного ПАВ распространяются до щелочной среды 
со значительным улучшением растворимости в нейтральной областй. Аналогичная ситуация наблюдается в случае соединения с одной дополнительной аминной группой $\mathrm{RCA}_{2}$. Здесь у соединения в нейтральной области сохраняются анионные свойства. Можно заключить, что свойства амфотерных ПАВ типа $\mathrm{RC}_{2} \mathrm{~A}$ и $\mathrm{RCA}_{2}$ отличаются от свойств амфотерных ПАВ типа RCA и мы вправе отнести их в отдельные, самостоятельные подклассы амфотерных ПАВ.
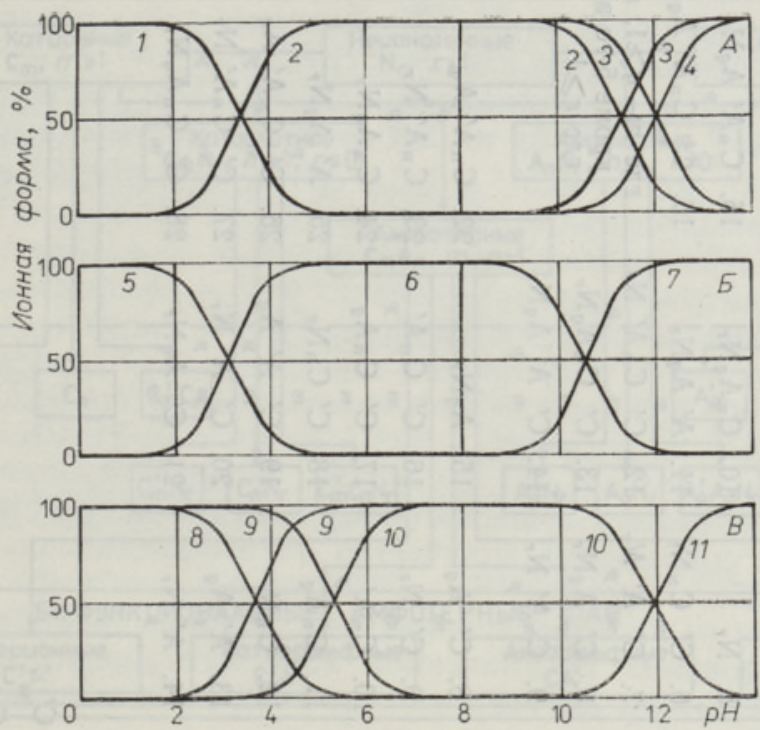

Рис. 3. Характеристики диссоциации амфотерных ПАВ.

A - тип $\mathrm{RC}_{2} \mathrm{~A}, \mathrm{C}_{8} \mathrm{H}_{17} \mathrm{NHCH}_{2} \mathrm{CH}_{2} \mathrm{NHCH}_{2} \mathrm{COOH}\left(\mathrm{pK}_{1}=2,03, \mathrm{pK}_{2}=8,74, \mathrm{pK}_{3}=10,36\left[{ }^{10}\right]\right)$, $1-\mathrm{R}_{8} \mathrm{C}_{2}^{2+} \mathrm{A}, 2-\mathrm{R}_{8} \mathrm{C}_{2}^{2+} \mathrm{A}^{-}, 3-\mathrm{R}_{8} \mathrm{C}^{+} \mathrm{CA}^{-}, 4-\mathrm{R}_{8} \mathrm{C}_{2} \mathrm{~A}^{-}$;

Б - Tun RCA, $\mathrm{C}_{12} \mathrm{H}_{25} \mathrm{NHCH}_{2} \mathrm{CH}_{2} \mathrm{COOH}\left(\mathrm{pK}_{1}=3,49, \mathrm{pK}_{2}=10,89\right.$ [ $\left.\left.{ }^{11}\right]\right), 5-\mathrm{R}_{12} \mathrm{C}+\mathrm{A}, 6-$ $\mathrm{R}_{12} \mathrm{C}^{+} \mathrm{A}^{-}, 7-\mathrm{R}_{12} \mathrm{CA}^{-}$;

$\mathrm{B}-$ тип $\mathrm{RCA}_{2}, \mathrm{C}_{8} \mathrm{H}_{17} \mathrm{~N}\left(\mathrm{CH}_{2} \mathrm{COOH}\right)_{2}\left(\mathrm{pK}_{1} \simeq 2\left[{ }^{7}\right], \mathrm{pK}_{2}=2,74, \mathrm{pK}_{3}=10,57\left[{ }^{12}\right]\right), 8-\mathrm{R}_{8} \mathrm{C}^{+} \mathrm{A}_{2}$, $9-\mathrm{R}_{8} \mathrm{C}^{+} \mathrm{AA}^{-}, 10-\mathrm{R}_{8} \mathrm{C}^{+} \mathrm{A}_{2}^{2-}, 11-\mathrm{R}_{8} \mathrm{CA}_{2}^{2-}$.

Учитывая указанные факторы, примем для группы однофункциональных и тех полифункциональных ПАВ, которые не содержат противоположно заряженных кислотных и основных групп, допущения, действующие в варианте классификации III (табл. 2). В результате образуются следующие классы и подклассы ПАВ (до ХД):

1. Катионные $-\mathrm{RC}_{m}$ или по ХД $-\mathrm{RC}_{m}^{\prime}, \mathrm{RC}_{n}$ и $\mathrm{RC}_{m}^{\prime} \mathrm{C}_{n}$, где $m, n \geqslant 1$.

2. Анионные $-\mathrm{R} \mathbf{A}_{n}$ или по ХД $-\mathrm{RA}_{p}^{\prime}, \mathrm{RA}_{q}$ и $\mathrm{RA}_{p}^{\prime} \mathrm{A}_{q}$, где $n$, $p, q \geqslant 1$.

3. Неионогенные - $\mathrm{RN}_{r}$, где $r \geqslant 1$.

4. Катионно-неионогенные (катиолитные ${ }^{*}$ ) $-\mathrm{RC}_{m} \mathrm{~N}_{r}$ или по ХД $\mathrm{RC}_{m}^{\prime} \mathrm{N}_{r}, \mathrm{RC}_{n} \mathrm{~N}_{r} \quad$ и $\mathrm{RC}_{m}^{\prime} \mathrm{C}_{n} \mathrm{~N}_{r}$, где $m, n, r \geqslant 1$.

5. Анионно-неионогенные (аниолитные ${ }^{*}$ ) - $\mathrm{RA}_{n} \mathrm{~N}_{r}$ или по ХД $\mathrm{RA}_{p}^{\prime} \mathrm{N}_{r}, \quad \mathrm{RA}_{q} \mathrm{~N}_{r} \quad$ и $\mathrm{RA}_{p}^{\prime} \mathrm{A}_{q} \mathrm{~N}_{r}$, где $p, q, r \geqslant 1$.

* Предлагаемые названия этих классов. 


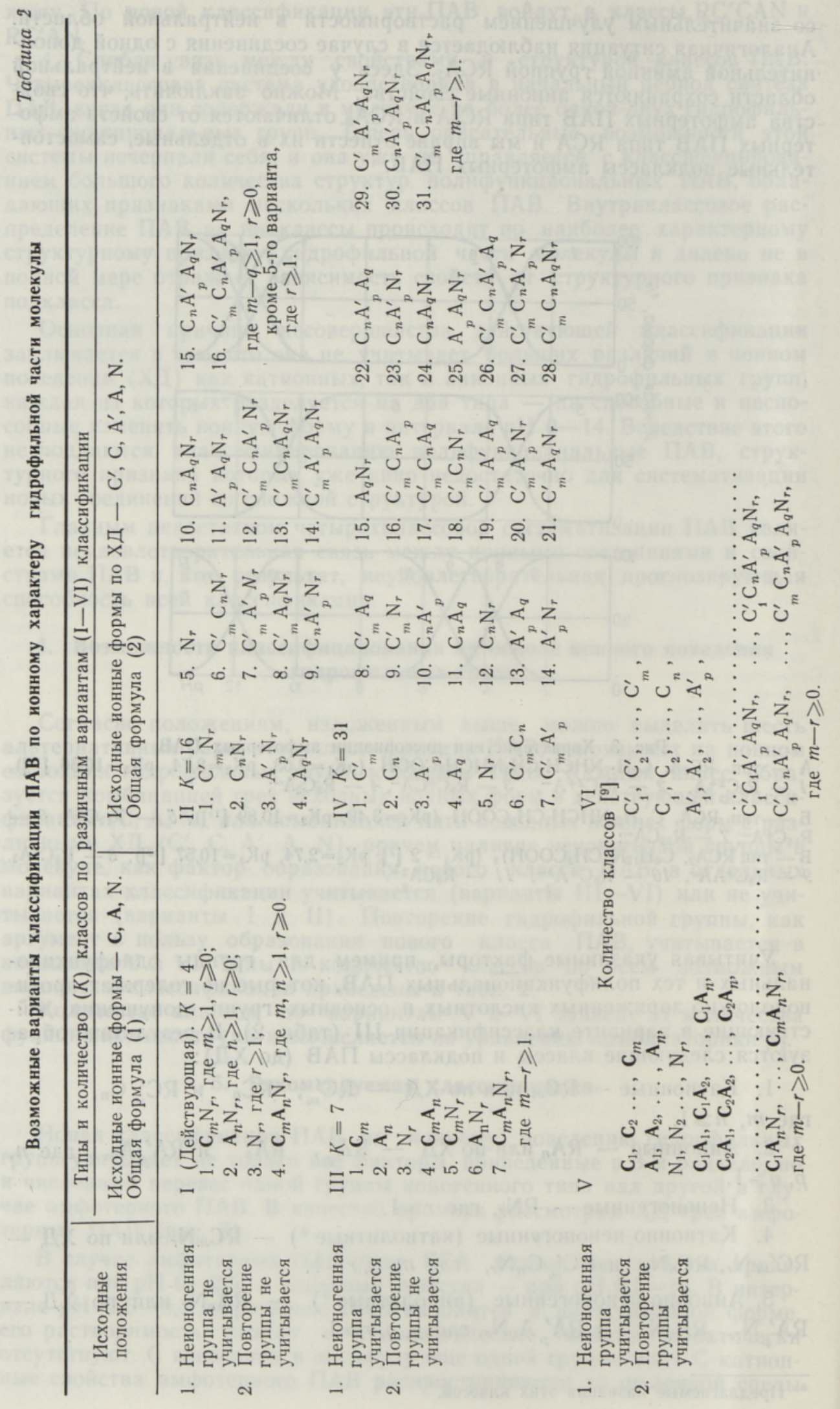


Из этих классов первые три признаны как самостоятельные, однако, классы ионогенных ПАВ могут по ХД быть разбиты на три подкласса. Классы, названные катислитными и аниолитными, являются новыми и сочетают в себе свойства катионных и неионогенных, анионных и неионогенных ПАВ соответственно. По ХД эти классы могут также быть разбиты на три подкласса (рис. 4).

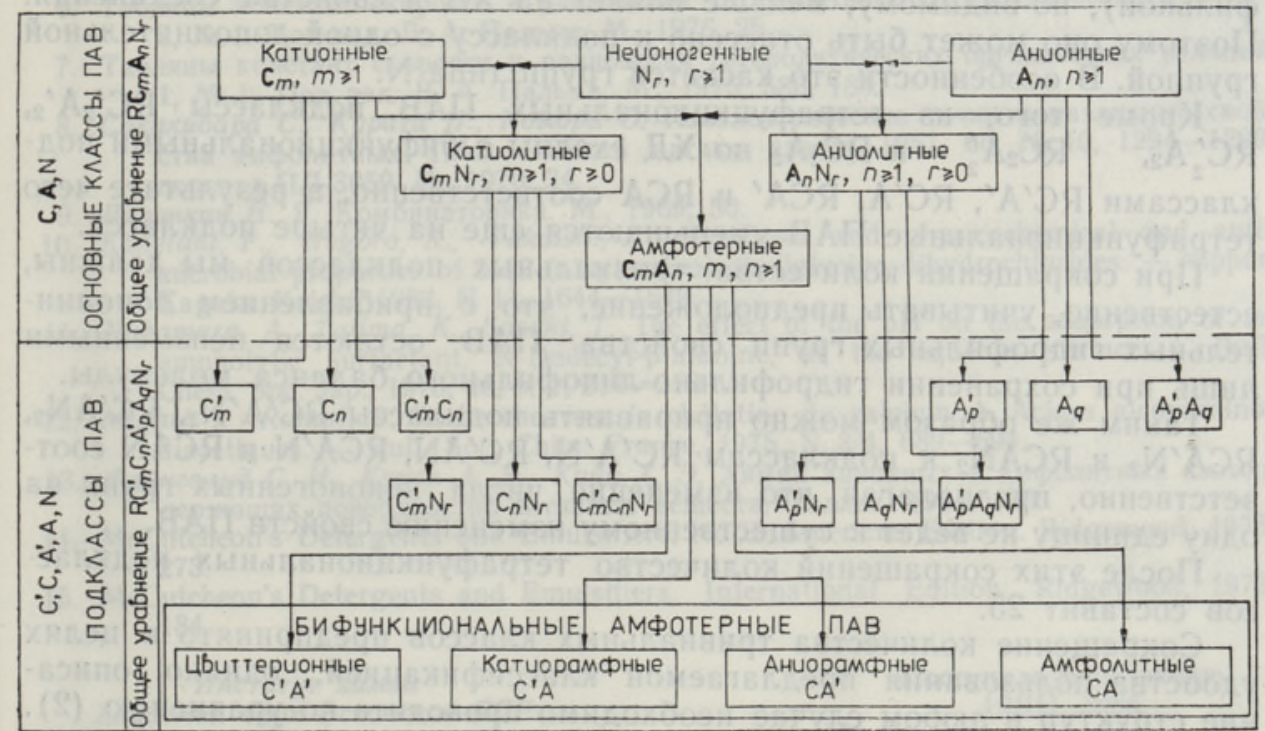

Рис. 4. Рекомендуемая классификация ПАВ.

Основные классы образованы на основе трех (C, A, N) типов гидрофильных групп, а подклассы - на основе пяти $\left(\mathrm{C}^{\prime}, \mathrm{C}, \mathrm{A}^{\prime}, \mathrm{A}, \mathrm{N}\right)$ типов (по характеристикам диссоциации).

Остальные ПАВ можно отнести к классу амфотерных ПАВ с коли чеством подклассов, образуемых по варианту классификации VI, т. е. с учетом ХД, наличия неионогенной группы и увеличения численного перевеса ионогенной группы как изменяющих свойства ПАВ факторов. Из этих подклассов бифункциональные амфотерные ПАВ можно разбить на четыре подкласса, трифункциональные на 16 (на рис. 4 не указаны), тетрафункциональные на 31 и т. д.

Схематически предлагаемая классификация представлена на рис. 4. Для описания ПАВ любой структуры может применяться формула (2), причем в этом случае необходимо располагать сведениями о константах ионизации гидрофильных групп.

О содержании названий «амфотерные», «цвиттерионные» и «амфолитные» ПАВ уже было сказано выше. Примененные нами названия «катиорамфные» и «аниорамфные» ПАВ (рис. 4) являются сокрашениями от «катионно-ориентированных» и «анионно-ориентированных» амфотерных ПАВ $\left.{ }^{13}\right]$. В первых совмещены группы типа $\mathrm{C}^{\prime}$ и A, во вторых - типа С и $\mathrm{A}^{\prime}$.

Образование трифункционального ПАВ прибавлением к бифункциональному дополнительной гидрофильной группы с другой ХД или с ХД, уже присущей молекуле, ведет к новым подклассам, пока безымянным. 
Их описание, как и менее сложных структур, можно провести посредством формулы (2). Например, соединение,

$$
\begin{array}{lccc}
\mathrm{C}_{12} \mathrm{H}_{25} \stackrel{+}{\mathrm{N}}\left(\mathrm{CH}_{2} \mathrm{CH}_{2} \mathrm{OH}\right)_{2} \mathrm{C}_{2} \mathrm{H}_{4} \mathrm{~N}\left(\mathrm{CH}_{3}\right) \mathrm{C}_{3} \mathrm{H}_{6} \mathrm{SO}_{3}^{-} \\
\mathrm{R}_{12} \mathrm{C}^{\prime} & \mathrm{N}_{2} & \mathrm{C} & \mathrm{A}^{\prime}
\end{array}
$$

является пятифункциональным и описывается формулой $\mathrm{R}_{12} \mathrm{C}^{\prime} \mathrm{CA}^{\prime} \mathrm{N}_{2}$.

Дальнейший избыток определенной группы (более одной гидро фильной), по-видимому, меньше влияет на ХД и свойства соединения. Поэтому оно может быть отнесено к подклассу с одной дополнительной группой. В особенности это касается групп типа N.

Кроме того, из тетрафункциональных ПАВ подклассы $\mathrm{RC}_{2} \mathrm{~A}_{2}$, $\mathrm{RC}_{2}^{\prime} \mathrm{A}_{2}, \quad \mathrm{RC}_{2} \mathrm{~A}_{2}^{\prime}$ и $\mathrm{RC}_{2} \mathrm{~A}_{2}$ по ХД сходны с бифункциональными подклассами $\mathrm{RC}^{\prime} \mathrm{A}^{\prime}, \mathrm{RC}^{\prime} \mathrm{A}, \mathrm{RCA}^{\prime}$ и $\mathrm{RCA}$ соответственно, в результате чего тетрафункциональные ПАВ уменьшаются еще на четыре подкласса.

При сокращении количества тривиальных подклассов мы должны, естественно, учитывать предположение, что с прибавлением дополнительных гидрофильных групп свойства ПАВ остаются неизменными лишь при сохранении гидрофильно-липофильного баланса молекулы.

Таким же образом можно приравнять подклассы $\mathrm{RC}^{\prime} \mathrm{A}^{\prime} \mathrm{N}_{2}, \mathrm{RC}^{\prime} \mathrm{AN}_{2}$, $\mathrm{RCA}^{\prime} \mathrm{N}_{2}$ и $\mathrm{RCAN}_{2}$ к подклассам RC'A'N, RC'AN, RCA'N и RCAN соответственно, предполагая, что изменение числа неионогенных групп на одну единицу не ведет к существенному изменению свойств ПАВ.

После этих сокращений количество тетрафункциональных подклас. сов составит 25 .

Сокращение количества тривиальных классов предпринято в целях удобства пользования предлагаемой классификацией, однако описание структур в любом случае необходимо проводить по уравнению (2).

\section{6. Методика обработки данных}

Результаты, представленные в табл. 1, получены обобщением данных, опубликованных в $\left[{ }^{14,15}\right]$.

В качестве исходного было принято положение, что о прикладных свойствах каждого конкретного соединения заявлено производителем или изобретателем.

Данные оценивались по двухбалльной системе $(0-$ свойство не заявлено, 1 - свойство заявлено). Баллы прикладных свойств всех coeдинений в каждом классе и подклассе ПАВ суммировались по формуле.

$$
F_{j}=\sum_{i=1}^{m} P_{i j}
$$

где $j=1, \ldots, n, F_{j}-$ сумма баллов прикладного свойства $P_{j}$ рассматриваемого ионного класса ПАВ, составленного множеством ПАВ, $i=1, \ldots, m$, и получали ряды прикладных свойств для каждого выделенного класса или подкласса ПАВ (табл. 1).

Полученные суммы указывают величины значимости каждого свойства внутри определенного класса или подкласса ПАВ при их практическом использовании, 
1. Mičič, , J. Klasifikacija tenzida, - Hemijska Industrija (Beograd), 1975, 29, N 8, $337-341$.

2. Поверхностно-активные вещества. Справочник. Л., 1979, 6-8.

3. Хидака Х. Амфотерные поверхностно-активные вещества. 1. Синтез. - Когё дзайрё, 1976, 24, № 5, 62-71; перевод ВЦП № А-57608. М., 1978, 29.

4. Абрамзон A. А. Структура поверхностно-активных веществ и классификация нх применения. - Ж. прикл. хим., 1980, 53, № 5, 1033-1040.

5. Blażej, A., Hodul, P., Markušouská, E., Novák, L., Paulovič, M., Vyskočil, I. Tenzidy. Bratislava-Praha, $1977,27-45$.

6. Таблицы констант скорости и равновесия гетеролитических органических реакций, 2, № 1. Под ред. В. А. Пальма. М., 1976, 25.

7. Таблицы констант скорости и равновесия гетеролитических органических реакций, 1, № 1. Под ред. В. А. Пальма. М., 1975, 580, 184.

8. Сакакибара С., Курата Н., Комори С. Изоэлектрические точки и различные свойства амфолитных ПАВ. - Когё кагаку дзасси, 1957, 60, № 10, 1294-1299; перевод ПЛ-3059. М., 1970, 24.

9. Валенкин Н. Я. Комбинаторика. М., 1969, 50.

10. Kanetani, F., Negoro, K., Takaishi, $H$. Synthesis and physicochemical and antimicrobial properties of $\mathrm{N}$-[2-(alkylamino) ethyl]glycine dihydrochlorides. - Nippon Kagaku Kaishi, 1983, N 11, 1644-1649.

11. Nakamura, A., Tajima, K., Sasaki, T. The effect of the $\mathrm{pH}$ on the adsorption of an ampholytic surfactant. N-Dodecyl- $\beta$-alanine, at the aqueous surface. - Bull. Chem. Soc. Jap., 1975, 48, N 1, 214-218.

12. Mighri, Z., Rumpf, P. Recherches sur la chélation du calcium, 1. Acides alcoyliminodiacétiques. - Bull. Soc. Chim. France, 1975, N 3/4, 689-694.

13. Файнгольд С. И., Кууск А. Э., Кийк Х. Э. Химня анионных и амфолитных азотсодержащих поверхностно-активных веществ. Таллин, 1984, 156.

14. McCutcheon's Detergents and Emulsifiers. North American Edition. Ridgewood, 1975, 273.

15. McCutcheon's Detergents and Emulsifiers. International Edition. Ridgewood, 1975, 184.
Институт химии
Академии наук Эстонской ССР
Поступила в редакцию
10/III 1986

\section{A. KUUSK}

\section{PINDAKTIIVSETE AINETE OMADUSTE SOLTUVUS NENDE KEEMILISEST STRUKTUURIST}

\section{Ioonklassifikatsioon}

On esitatud ioonklassifikatsioon, mis olemasolevaga vōrreldes kirjeldab täpsemini pindaktiivsete ainete ioonilist käitumist. On kasutatud uut põhimõtet struktuuri (ioonklasside) ja omaduste (rakendusomadused) seose analüüsiks. Uutele ja ka vanadele ioonklassidele on kirjandusandmete läbitöötamisel leitud rakendusomaduste pingeread, mille abil saab mõningase tōenäosusega prognoosida mis tahes etteantud struktuuriga pind-
aktiivsete ainete omadusi.

\section{A. KUUSK}

\section{STRUCTURE-PROPERTY RELATIONSHIP OF SURFACTANTS}

\section{Ionic classification}

At the present time all surfactants are observed as consisting of three types of hydrophilic groups: cationic (C), anionic (A) and nonionic (N). Consequently, all surfactants
may be presented by the formula

$$
\mathrm{RC}_{m} \mathbf{A}_{n} \mathrm{~N}_{r}
$$

whẹre $m, n, r \geqslant 0$; R - hydrophobic moiety of molecules, 
It was proposed to divide ionogenic groups into two types - those capable within the $\mathrm{pH}$ range $0-14$ by protolysis of completely turning into another ionic form and those having within this range constant ionic charge. Consequently, all surfactants may be expressed more exactly by the formula

$$
\mathrm{RC}_{m}^{\prime} \mathrm{C}_{n} \mathrm{~A}_{p}^{\prime} \mathrm{A}_{q} \mathrm{~N}_{r},
$$

where $m-r \geqslant 0 ; \mathrm{C}^{\prime}$ and $\mathrm{A}^{\prime}-$ ionogenic groups having within the $\mathrm{pH}$ range $0-14$ a constant ionic charge; $\mathrm{C}$ and $\mathrm{A}$ - ionogenic groups capable within this range of turning into another ionic form.

If we determine $\mathrm{pH}$ dependence of ionic form types as a dissociation characterictics (DC), then we may classify all surfactants by DC into ionic classes. The surfactants with an identical shape of DC formed independent classes.

The applied properties of surfactants correlated with the ionic classes and for each class the order of applied properties is established. These orders allow the properties of any surfactant to be predicted with some probability. 